ARTICLE

Received 1 Feb 2017 | Accepted 23 May 2017 | Published 20 Jul $2017 \quad$ DOl: 10.1038/ncomms16089 OPEN

\title{
Strain-induced skeletal rearrangement of a polycyclic aromatic hydrocarbon on a copper surface
}

Akitoshi Shiotari ${ }^{1}$, Takahiro Nakae ${ }^{2}$, Kota Iwata ${ }^{3}$, Shigeki Mori ${ }^{4}$, Tetsuo Okujima ${ }^{5}$, Hidemitsu Uno ${ }^{5}$, Hiroshi Sakaguchi ${ }^{2} \&$ Yoshiaki Sugimoto ${ }^{1,3}$

Controlling the structural deformation of organic molecules can drive unique reactions that cannot be induced only by thermal, optical or electrochemical procedures. However, in conventional organic synthesis, including mechanochemical procedures, it is difficult to control skeletal rearrangement in polycyclic aromatic hydrocarbons (PAHs). Here, we demonstrate a reaction scheme for the skeletal rearrangement of PAHs on a metal surface using high-resolution noncontact atomic force microscopy. By a combination of organic synthesis and on-surface cyclodehydrogenation, we produce a well-designed PAH-diazuleno[1,2,3-cd:1', $\left.2^{\prime}, 3^{\prime}-f g\right]$ pyrene-adsorbed flatly onto $\mathrm{Cu}(001)$, in which two azuleno moieties are highly strained by their mutual proximity. This local strain drives the rearrangement of one of the azuleno moieties into a fulvaleno moiety, which has never been reported so far. Our proposed thermally driven, strain-induced synthesis on surfaces will pave the way for the production of a new class of nanocarbon materials that conventional synthetic techniques cannot attain.

\footnotetext{
${ }^{1}$ Department of Advanced Materials Science, The University of Tokyo, 277-8561 Kashiwa, Japan. ${ }^{2}$ Institute of Advanced Energy, Kyoto University, 611-0011 Uji, Japan. ${ }^{3}$ Graduate School of Engineering, Osaka University, 565-0871 Suita, Japan. ${ }^{4}$ Advanced Research Support Center, Ehime University, 790-8577 Matsuyama, Japan. ${ }^{5}$ Graduate School of Science and Engineering, Ehime University, 790-8577 Matsuyama, Japan. Correspondence and requests for materials should be addressed to A.S. (email: shiotari@k.u-tokyo.ac.jp) or to T.N. (email: nakae.takahiro.8m@kyoto-u.ac.jp).
} 
S tructural strains in organic molecules play a huge role in determining molecular stability. Steric repulsions between component atoms in a hydrocarbon molecule cause the distortion of orbital geometries (such as $s p^{3}$ and $s p^{2}$ hybridizations) of carbon atoms, giving rise to characteristic reactivity in order to release the strain energy. For instance, the Baeyer strain ${ }^{1}$, an angle strain of $s p^{3}$-hydrocarbon rings, is responsible for the ring-opening reactivity of bicyclic compounds ${ }^{2}$. A structural strain can also be applied to molecules by external stimuli; organic synthesis promoted by stress added to reactants is categorized as mechanochemistry ${ }^{3-5}$. Mechanical stimuli, such as high-pressure conditions and ultrasound exposure, can modify molecular geometries to yield specific products that cannot be induced by heat, light or electric fields ${ }^{6}$.

Strain-induced rearrangements of aromatic carbon rings have been observed in graphene structures. As a typical example, a mechanical stress in graphene triggers a Stone-Wales rearrangement ${ }^{7,8}$, a typical reaction of carbon allotropes that yields a defect composed of two heptagonal and two pentagonal rings fused together. Such defects play an important role in the formation of curved graphene and fullerenes ${ }^{8-12}$. Therefore, it is crucial to control the rearrangements of $s p^{2}$-carbon skeletal structures in order to synthesize nanocarbon materials ${ }^{13-15}$. For macromolecules such as graphene, intramolecular rearrangements feasibly occur because such local reactions do not strictly affect the total energy of the huge systems. For small polycyclic aromatic hydrocarbons (PAHs), on the other hand, it has been difficult to induce skeletal rearrangements in conventional organic synthesis. The preceding mechanochemical procedures for aromatic compounds cannot induce unimolecular reactions but can induce $s p^{3}$-hybridized polymerizations ${ }^{16,17}$ because of the enormous increase in intermolecular interactions. Other synthetic methods under severe conditions, such as flash vacuum pyrolysis ${ }^{13,18}$, are required to undermine the aromaticity in the transition states and to obtain intramolecularly rearranged PAHs. Therefore, the exploration of PAH reaction schemes will be a significant step towards understanding the mechanisms underlying the creation and repair of defects in carbon allotropes, and towards designing and fabricating further functional nanocarbon materials.

It is well known that the adsorption of organic molecules onto metal surfaces modifies the molecular geometries and electronic states ${ }^{19,20}$. Adsorbate-substrate interactions can promote characteristic thermal reactions (namely, on-surface synthesis ${ }^{21,22}$ ) occurring in neither the crystal nor the solution, which can yield well-defined products such as graphene nanoribbons $s^{23-26}$ and other nanocarbons ${ }^{27-31}$. Therefore, we conceive that the geometric structure of a PAH can be distorted by adsorption onto a metal surface, so that the molecule may store strain energy to facilitate a catalytic reaction at the surface. Although some previous studies have implied that the structural distortion of precursors on surfaces is a key factor for on-surface reactions ${ }^{24,26,28,31}$, the correlation between the structural strain and reactivity has not been focused on in the previous studies and remains less well understood. Besides, the binding of a target molecule to a flat surface provides an overwhelming advantage in the characterization of individual molecules on the surface with scanning tunnelling microscopy (STM) and atomic force microscopy (AFM). In particular, noncontact AFM with a functionalized tip (that is, a probe attaching an atom or a molecule to its apex) can visualize chemical bonding structures of adosorbates ${ }^{32-37}$, because it can sensitively detect the repulsive forces between atoms in the adsorbate and the sharpened tip ape ${ }^{38,39}$. By using this imaging technique, reactants, intermediates and products of various on-surface reactions have successfully been identified ${ }^{31,40-46}$. We focus on the on-surface reactivity of azulene-derivative PAHs. Azulene $\left(\mathrm{C}_{10} \mathrm{H}_{8}\right)$, a typical

nonbenzenoid aromatic compound composed of seven- and five-membered rings, can be thermally rearranged into its structural isomer of naphthalene $e^{47,48}$. Such rearrangement of an azulene-type skeletal structure in PAHs is strongly related to the Stone-Wales defects mentioned above $\mathrm{e}^{7,8,49,50}$. Despite the importance of understanding the reactivity, studies on azulene and its derivatives adsorbed onto surfaces have been scarce ${ }^{51,52}$.

Here we show thermally driven intramolecular rearrangement with the assistance of a structural strain of $s p^{2}$-aromatic rings. We utilize a metal surface as a tight-binding field to generate a structural strain in the $\mathrm{PAH}$ due to the deformation of the molecular structure, a reaction field to catalytically activate intermolecular rearrangement of the $\mathrm{PAH}$, and an observation field to identify the individual reaction products. We newly synthesize geometrically isomeric PAHs with two azuleno moieties. We observe these molecules adsorbed onto a metal surface with STM and noncontact AFM, and compared the difference in reactivity between the PAHs. We verify that the intramolecular structural strain drives the catalytic reaction to rearrange the azuleno moiety.

\section{Results}

Adsorption of intrinsically distorted PAHs onto $\mathbf{C u ( 0 0 1 )}$. We synthesized two kinds of PAHs: diazuleno[1,2-c:2', $\left.1^{\prime}-g\right]$ phenanthrene (DAPh) 1 (Fig. 1a) and diazuleno $\left[1,2-a: 2^{\prime}, 1^{\prime}-c\right]$ anthracene (DAA) 2 (Fig. 1b). The former has a highly twisted geometry in free space because of the steric repulsion between two $\mathrm{H}$ atoms
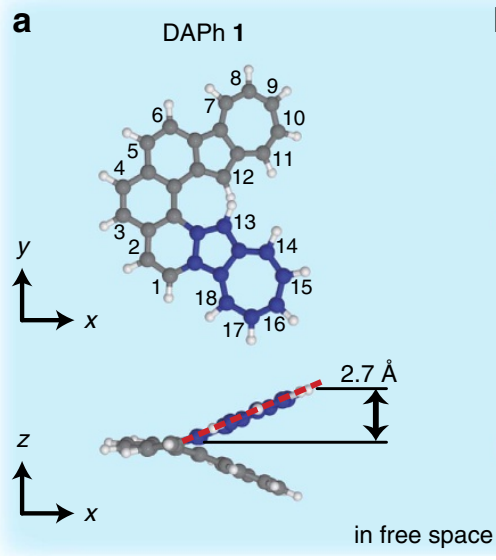

C
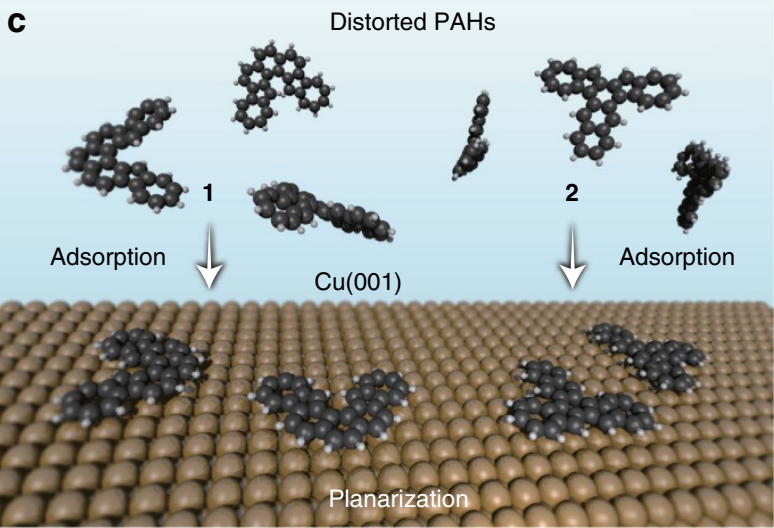

Figure 1 | Molecular structures of synthesized azulene-derivative PAHs. (a,b) Calculated structures of diazuleno[1,2-c:2', $\left.1^{\prime}-g\right]$ phenanthrene (DAPh) 1 and diazuleno[1,2- $\left.a: 2^{\prime}, 1^{\prime}-c\right]$ anthracene (DAA) 2, respectively, in free space. Numbers at carbon atoms were assigned according to the IUPAC nomenclature. Blue spheres in a represent $C$ atoms of one of the azuleno moieties in DAPh 1. (c) Scheme of the adsorption of $\mathbf{1}$ and $\mathbf{2}$ onto a Cu(001) surface to modify the molecular geometries. 
bonded to the 12- and 13-positioned $\mathrm{C}$ atoms in the azuleno moieties ( $C_{2}$ point group; Fig. 1a). The latter is a structural isomer of $\mathrm{DAPh}$ with a different steric structure: a bent geometry in free space ( $C_{\mathrm{s}}$ point group) because of the $\mathrm{H}-\mathrm{H}$ repulsions between the 5 and 6 positions and between the 17 and 18 positions. We note that this molecule forms a twisted geometry in the crystal phase $\left(C_{2}\right.$ point group; see Supplementary Data 2$)$. Our calculations of DAA in free space show that the bent configuration is $113 \mathrm{meV}$ more stable than the twisted one. Here we define the molecular height as the distance between the highest- and lowest$z$ atoms when the phenanthrene/anthracene part of the molecule is placed in an $x-y$ plane. The height of the free DAPh is calculated to be $5.4 \AA$, indicating that each azuleno moiety is distorted with a height of $2.7 \AA$ (Fig. 1a). Because the free DAA has a molecular height of $2.6 \AA$ (Fig. 1b), the azuleno moieties in both DAPh and DAA molecules are distorted in a similar manner. We target the intrinsically distorted molecules as reactants, because adsorption of the molecules onto a surface is potentially capable of modifying the geometry.

Each isomer we synthesized was adsorbed to a $\mathrm{Cu}(001)$ surface (Fig. 1c) and the molecular geometries on the surface were elucidated by using STM/AFM. Figure 2a,b shows topographic STM images of DAPh 1 and DAA 2, respectively, on the surface at $4.8 \mathrm{~K}$. We used CO-terminal tips ${ }^{32}$ in order to achieve higher spatial resolution in the STM and AFM images (see Supplementary Note 1 and Supplementary Fig. 1). With STM, DAPh (DAA) is imaged as a symmetric V- (T-) shaped protrusion. Figure $2 \mathrm{c}, \mathrm{d}$ shows AFM images of DAPh and DAA, respectively. In both AFM images, all covalent bonds of the molecule are visualized due to the detection of the repulsive forces between atoms in the adsorbate and $\mathrm{CO}$ attached to the tip apex. $\mathrm{C}-\mathrm{C}$ bonds at the molecular edges are seemingly emphasized because the long-range attractive forces that appear as blurred depressions in the images contribute predominantly at the molecular centre $\mathrm{e}^{32}$. It is well known that a frequency-shift $(\Delta f)$ contrast depends strictly on the atom height with respect to the surface; if a molecule is not flat but twisted or bent, the $\Delta f$ signals in the sterically protruded region in the molecule must be much larger (that is, more repulsive against the probe tip $)^{31,41,46,53-55}$. In the images of DAPh and DAA, on the other hand, all covalent bonds within the molecules are clearly resolved, suggesting that both of the intrinsically distorted PAHs adsorbed flatly onto the surface.

The adsorption sites of these molecules were experimentally determined by STM images at a higher tunnelling current where the molecule and surface $\mathrm{Cu}$ atoms are observed simultaneously (Supplementary Note 1 and Supplementary Fig. 2). The adsorption sites of DAPh and DAA are shown in Fig. 2e,f, respectively. Both adsorption structures belong to the $C_{\mathrm{s}}$ point group with a symmetry axis along the $\langle 110\rangle$ direction. This supports the conclusion that these molecules are flattened on the surface. The modification of the steric structures suggests that the interaction between the molecule and the substrate was strong enough to overcome the geometric distortion of the flattened molecules. According to the adsorption energies of PAHs on $\mathrm{Cu}$ surfaces in the literature ${ }^{20}$, the adsorption energies of DAPh and DAA on $\mathrm{Cu}(001)$ are estimated to be roughly $4 \mathrm{eV}$. The flattening energies (that is, the energy between the optimized structure and the geometry that has been totally flattened in free space) of the molecules are calculated to be much lower than the estimated adsorption energies (Table 1). The interaction between all of the component atoms in the flattened molecules and the substrate stabilizes the systems.

On-surface reactivity of DAPh and DAA. Next we focus on the thermal reactivity of $\mathrm{DAPh}$ and DAA molecules on $\mathrm{Cu}(001)$.
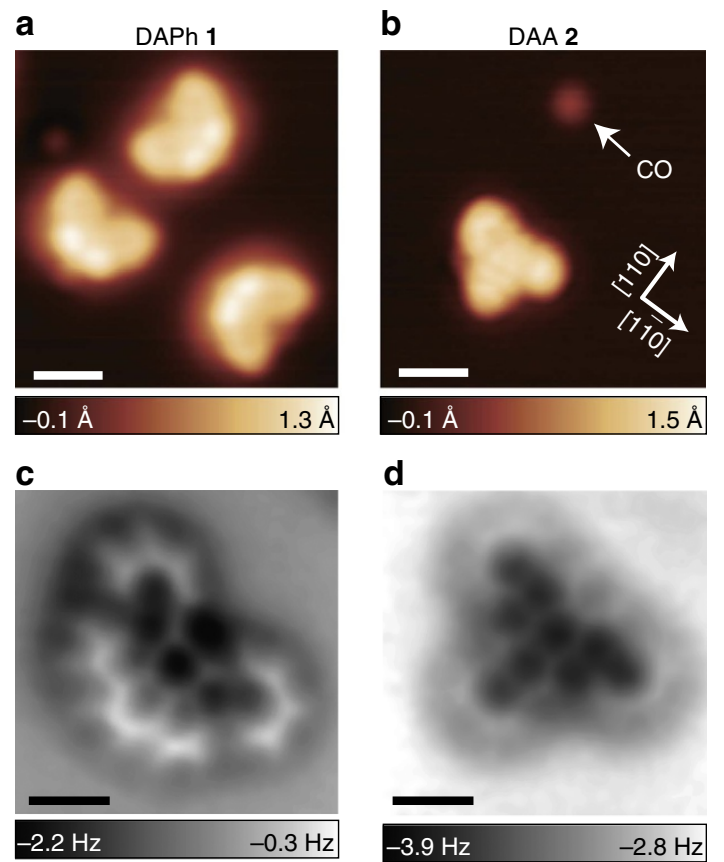

d
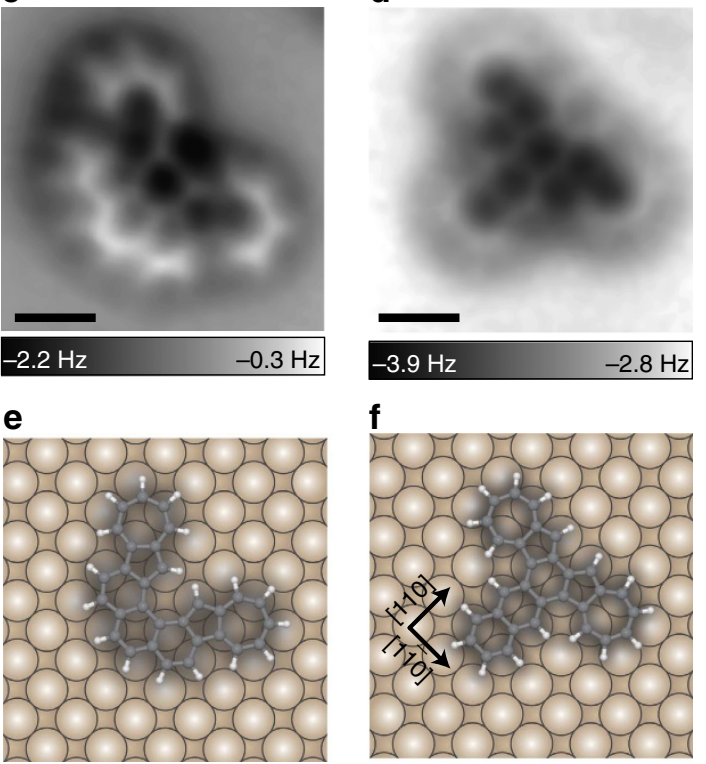

Figure 2 | AFM images and adsorption structures of DAPh and DAA on Cu(001). (a,b) Typical STM images of DAPh $\mathbf{1}$ and DAA 2, respectively, on $\mathrm{Cu}(001)$ at $4.8 \mathrm{~K}$ with CO-terminal tips. (c,d) AFM images of $\mathbf{1}$ and $\mathbf{2}$, respectively. (e,f) Schematics of the adsorption structures of $\mathbf{1}$ and $\mathbf{2}$, respectively. The nonplanar $\mathrm{PAHs}$ are expected to be flattened on the surface. The adsorption sites were determined experimentally (see Supplementary Fig. 2). The image in a was obtained with the sample bias $V=-30 \mathrm{mV}$ and the tunnelling current $I=10 \mathrm{pA}$. The image in $\mathbf{b}$ was obtained with $V=30 \mathrm{mV}$ and $I=20 \mathrm{pA}$. The image in $\mathbf{c}, \mathbf{d}$ was acquired at the tip height corresponding to $V=50 \mathrm{mV}$ and $I=200$ (20) pA over a bare surface. Scale bars, $10 \AA$ (a,b); $5 \AA$ (c,d).

A sample was annealed and kept at a desired temperature $T_{\text {anneal }}$ for $10 \mathrm{~min}$, followed by STM/AFM observation at $4.8 \mathrm{~K}$. After the measurements, the sample was cleaned and exposed to the molecular vapour at room temperature again to prepare a sample for another $T_{\text {anneal }}$. The coverage of the molecules varied between $1-7 \times 10^{12} \mathrm{~cm}^{-2}$ during the preparation-and-annealing cycles, but no coverage dependence was observed. For DAA 2, almost none of the adsorbates on $\mathrm{Cu}(001)$ reacted and no specific product was observed (see Supplementary Note 2 and Supplementary Fig. 3) even after annealing at $T_{\text {anneal }}=165-275^{\circ} \mathrm{C}$. On the other hand, several kinds of products were observed in the postannealed sample of $\mathrm{DAPh} / \mathrm{Cu}(001)$. As shown in Fig. 3d, the production yields depended on $T_{\text {anneal }}$. The pristine DAPh molecules 1 (Fig. 2a) were observed predominantly below $T_{\text {anneal }}=110^{\circ} \mathrm{C}$, whereas the yield of another species, 3 , increases at $T_{\text {anneal }}=125^{\circ} \mathrm{C}$ (Fig. 3a). As shown in Fig. 3a, 3 is clearly characterized by its STM image having a round protrusion near the molecular centre. The chemical assignment of the species is discussed below. Above $T_{\text {anneal }} \approx 130^{\circ} \mathrm{C}$, the yield of 3 decreases 
Table 1 | Calculated flattening energies in free space.

\begin{tabular}{|c|c|c|c|c|c|}
\hline & DAPh 1 & DAA 2 & DAPyr 4 & Fulvaleno 5 & Naphto 6 \\
\hline$\overline{\Delta G\left(25^{\circ} \mathrm{C}\right)}$ & 0.014 & 0.045 & 0.098 & 0.000 & 0.058 \\
\hline$\Delta G\left(227^{\circ} \mathrm{C}\right)$ & 0.060 & 0.104 & 0.171 & 0.000 & 0.110 \\
\hline
\end{tabular}

The energies are calculated in eV. $\Delta G(T)$ represents the Gibbs energy difference between the free molecule $G_{\text {free }}$ and the planar molecule (located in the $x-y$ plane with $z$ restricted to zero) $G_{\text {planar }}$ at a temperature $T$, that is, $\Delta G=G_{\text {planar }}-G_{\text {free }}$.

a

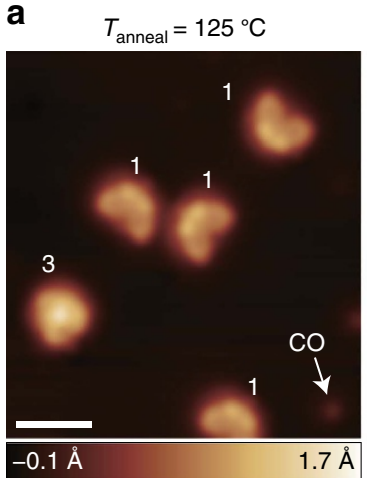

$-0.1 \AA$ b

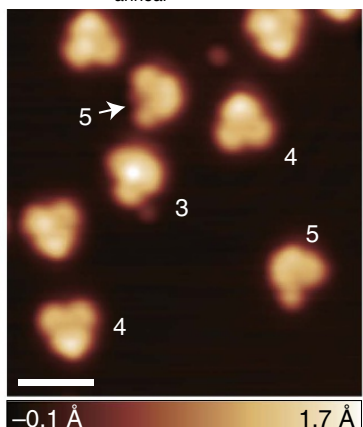

C

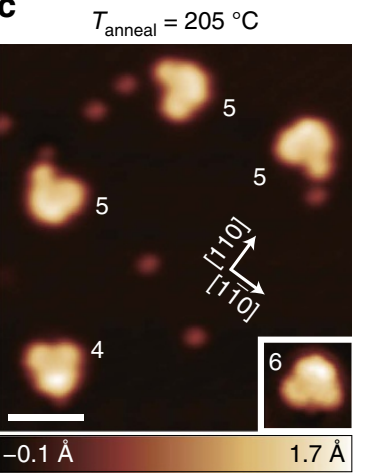

d

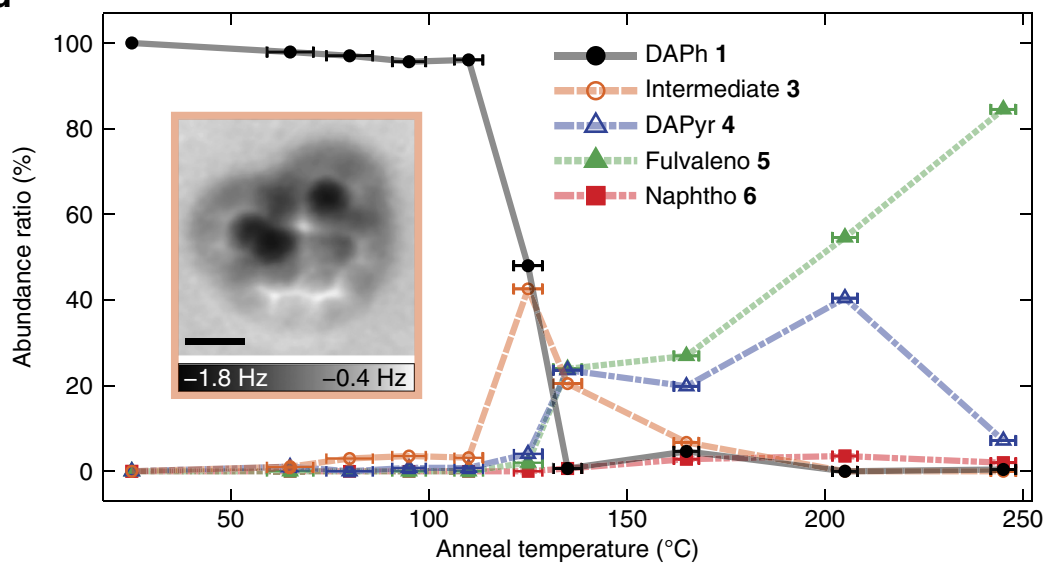

e
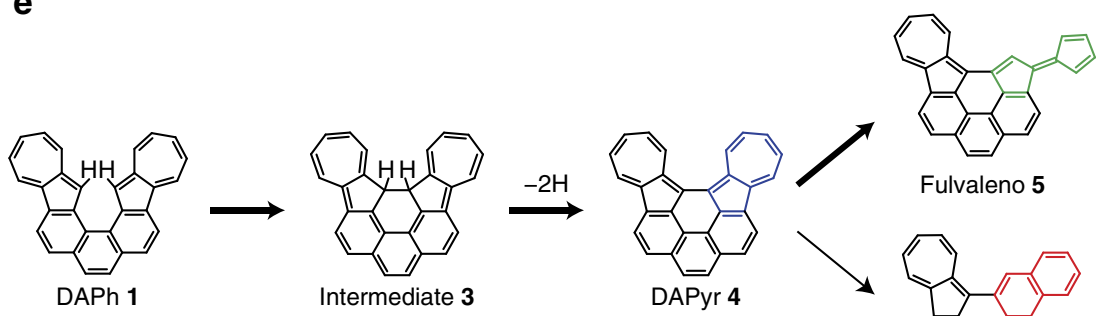

Fulvaleno 5

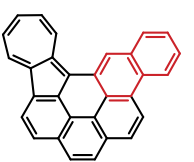

Naphtho 6

Figure 3 | Thermal reaction of DAPh on Cu(001). (a-c) Typical STM images of DAPh/Cu(001) at $4.8 \mathrm{~K}$ with $\mathrm{CO}$ terminal tips after annealing for 10 min at 125,135 and $205^{\circ} \mathrm{C}$, respectively. The inset of $\mathbf{c}$ shows an STM image of a minor product $\mathbf{6}$. (d) Annealing-temperature dependence of the abundance ratio of the molecular species on the surface. More than 100 molecules were counted at each annealing temperature. The error bar represents the standard deviation from the set-point temperature. The inset of $\mathbf{d}$ shows an AFM image of intermediate $\mathbf{3}$. (e) Reaction of DAPh $\mathbf{1}$ on Cu(001) to yield a fulvalenorearranged product $\mathbf{5}$ and a naphtho-rearranged product $\mathbf{6}$ via intermediates $\mathbf{3}$ and $\mathbf{4}$. The images were obtained with $V=-30 \mathrm{mV}$ and $I=10 \mathrm{pA}$ for $\mathbf{a}$, with $V=50 \mathrm{mV}$ and $I=20 \mathrm{pA}$ for $\mathbf{b}$ and the inset of $\mathbf{d}$, and with $V=50 \mathrm{mV}$ and $I=200 \mathrm{pA}$ for $\mathbf{c}$. The image in the inset of $\mathbf{d}$ was acquired at the tip height corresponding to $V=50 \mathrm{mV}$ and $I=20 \mathrm{pA}$ over a bare surface. Scale bars, $20 \AA$ (a-c); $5 \AA$ (the inset of d).

gradually with an increase in the abundance ratios of other images, namely, products 4 and 5 (Fig. 3d). With STM, 4 is imaged as a symmetric $\mathrm{V}$-shaped protrusion oriented to the $<100>$ direction, whereas $\mathbf{5}$ is observed as an asymmetric
$\mathrm{V}$-shaped protrusion (Fig. 3b). Figure $3 \mathrm{c}$ shows an STM image of the sample at $T_{\text {anneal }}=205^{\circ} \mathrm{C}$, where 3 was not observed but 4 and 5 existed predominantly. Above $T_{\text {anneal }}=165^{\circ} \mathrm{C}$, we also observed a very minor species 6 (inset of Fig. $3 \mathrm{c}$ ), with abundance 

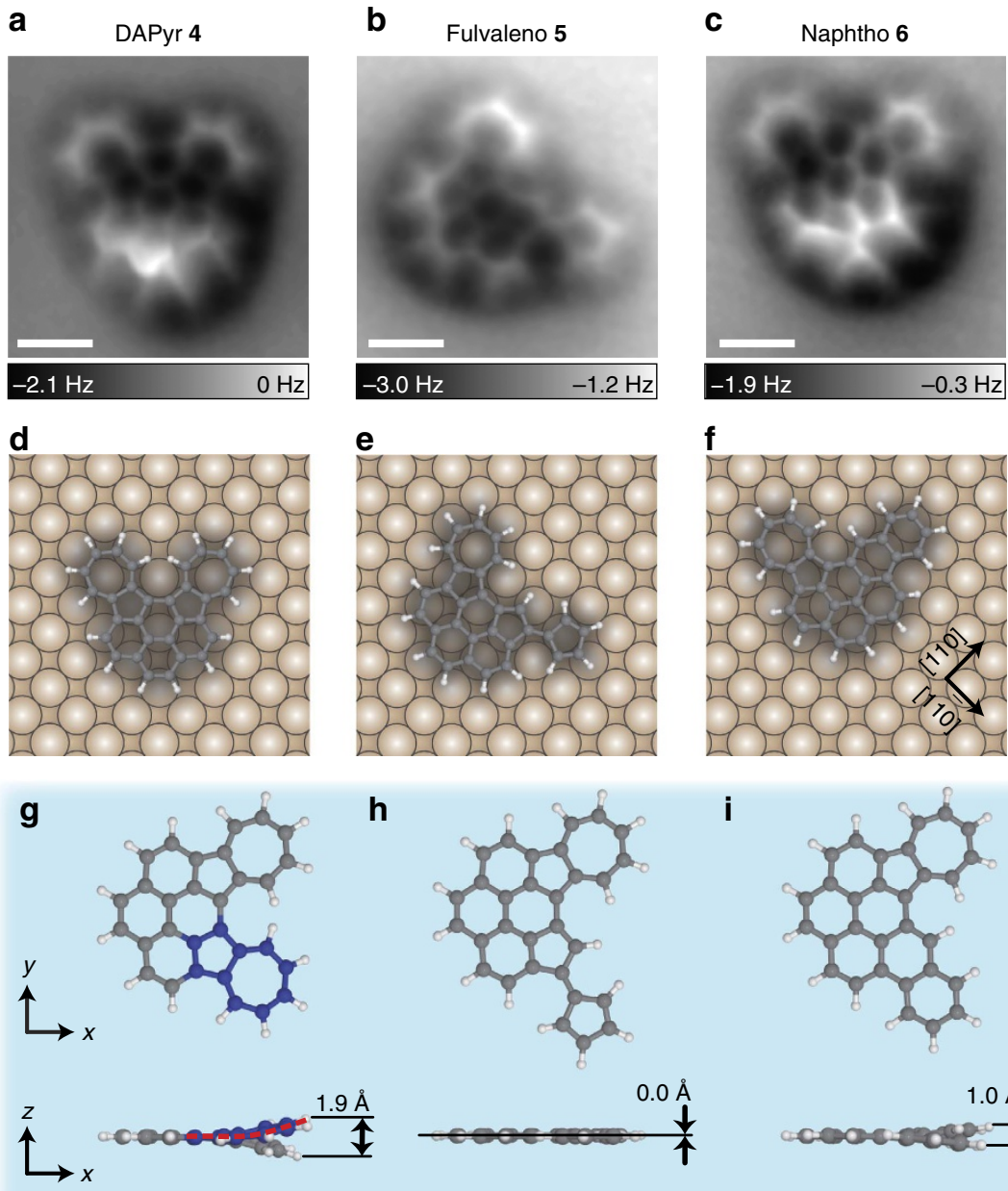

h

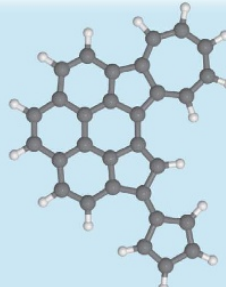

i

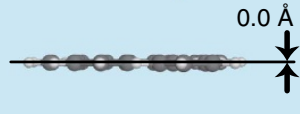

in free space

Figure 4 | AFM images of the thermal reaction products of DAPh/Cu(001). (a-c) AFM images of 4, $\mathbf{5}$ and $\mathbf{6}$, respectively, on Cu(001) in constant-height mode with CO-terminal tips. (d-f) Schematic illustration of the adsorption structures of $\mathbf{4}, \mathbf{5}$ and $\mathbf{6}$, respectively. All of the products are expected to be flattened on the surface. ( $\mathbf{g}-\mathbf{i})$ Calculated structures of $\mathbf{4}, \mathbf{5}$ and $\mathbf{6}$, respectively, in free space. Blue spheres in $\mathbf{g}$ represent $\mathbf{C}$ atoms of one of the azuleno moieties in DAPyr $\mathbf{4}$. A bent red line in $\mathbf{g}$ indicates that the sterically hindered azuleno moieties are distorted in free space. The images in a-c were measured at the tip height corresponding to $V=50 \mathrm{mV}$ and $I=200 \mathrm{pA}$ over a bare surface. Scale bar, $5 \AA$.

ratios of not more than $3 \% .5$ became predominant at $T_{\text {anneal }}=245{ }^{\circ} \mathrm{C}$, but finally, no molecule was observed at $T_{\text {anneal }}=300^{\circ} \mathrm{C}$ because all molecules were desorbed from the surface. Figure $3 \mathrm{~d}$ shows the annealing-temperature dependence of the abundance ratio for $1,3-6$. Above $125^{\circ} \mathrm{C}$, the sum of the ratios for the five species is not equal to $100 \%$ because several kinds of other species (minor products, intermediates and impurities) were also observed (see Supplementary Note 3 and Supplementary Fig. 4). According to the abundance ratios in Fig. 3d, we assume that the thermal reaction pathway of DAPh on the surface is $\mathbf{1} \rightarrow \mathbf{3} \rightarrow \mathbf{4} \rightarrow \mathbf{5 / 6}$. Note that this is a tentative assignment and that multiple pathways, for example, $\mathbf{4} \rightarrow \mathbf{6} \rightarrow \mathbf{5}$, might be possible.

High-resolution AFM imaging with CO-terminal tips is a useful technique for identifying the structure of the reaction intermediates and products. Figure 4a-c shows AFM images of 4-6, respectively. As with $\mathbf{1}$ (Fig. 2c), all of the $\mathrm{C}-\mathrm{C}$ bonds in each molecule are visible, suggesting that products 4-6 lie on the surface flatly. The AFM images determine the molecular structures and the reaction pathway as shown in Fig. 3e. The intermediate 4 , observed at $T_{\text {anneal }} \approx 130-210^{\circ} \mathrm{C}$, is assigned to a cyclodehydrogenated DAPh, namely diazuleno[1,2,3-cd: $\left.: 1^{\prime}, 2^{\prime}, 3^{\prime}-f g\right]$ pyrene (DAPyr; see Fig. 4d). Figure 4b,c shows AFM images of 5 and 6, respectively. The image indicates obviously that $\mathbf{5}(\mathbf{6})$ is yielded by the rearrangement of one of the two azuleno moieties in DAPyr 4 into a fulvaleno (naphtho) moiety as shown in Fig. 4 e,f.

\section{Discussion}

As stated above, DAPh 1 on the surface shows (i) cyclodehydrogenation at $\sim 130^{\circ} \mathrm{C}$, followed by (ii) the skeletal rearrangement above $200^{\circ} \mathrm{C}$, whereas DAA 2 is nonreactive. First, we consider the dehydrogenation mechanism. Although both DAPh $\mathbf{1}$ and DAA 2 are similarly distorted in free space (Fig. 1a,b), the sterically repelled $\mathrm{H}$ atoms of the former molecule can be dissociated thermally on the surface. The flattening energy of DAPh 1 is rather smaller than that of DAA 2 (Table 1), suggesting that the structural distortion does not directly contribute to this reaction. The reactivity difference is probably ascribable to the formability of the additional $\mathrm{C}-\mathrm{C}$ covalent bonding. If DAA 2 was cyclodehydrogenated, the 5- and 6-positioned C atoms (Fig. 1b) would be bonded covalently to form a new pentagonal ring; however, this product is significantly unstable because the $s p^{2}$-carbon skeleton is highly deformed. DAPh $\mathbf{1}$ is cyclodehydrogenated sensibly to form an additional 
hexagonal ring, but this reaction creates a large intramolecular distortion on the surface. The cyclodehydrogenated productDAPyr 4-is intrinsically twisted in free space because of steric hindrance between the azuleno moieties (Fig. 4g). As compared to DAPh 1 in which the phenanthrene part is twisted but the azuleno moieties are planar themselves (blue spheres aligning on a linear red line in Fig. 1a), the azuleno moieties of DAPyr 4 are distorted in free space (blue spheres aligning on a bent line in Fig. $4 \mathrm{~g}$ ) because of the rigid structure of pyrene part. Notably, for DAPyr 4, the new six-membered carbon ring in the pyrene part has no aromatic feature, which was confirmed by calculations of nuclear independent chemical shift for the free molecules (see Supplementary Fig. 5). Therefore, this cyclodehydrogenated reaction does not provide the extension of the aromatic system. DAPyr 4 is flattened on the surface (Fig. 4d), and then, the distortion of the azuleno moieties gives rise to larger structural strain than that of the reactant $\mathbf{1}$ (Table 1). We assume that the structural strain localized at the azuleno moieties drives the skeletal rearrangement of $\mathbf{4}$, as discussed below.

Species 3, which appears before yielding 4, probably intermediates the cyclodehydrogenated reaction from 1 to 4 . An AFM image of 3 (inset of Fig. 3d) shows an asymmetric outline with a high-contrast (that is, strongly repulsive) region located near the molecular centre. The sterically protruding structure of 3 complicates the determination of the detailed structure only from this image. We tentatively ascribed the intermediate to a DAPh with a C-C bond between the 12 and 13 positions without dehydrogenation (Fig. 3e). This molecule probably retains its aromaticity (26 $\pi$ electrons) but is metastable because of the extremely distorted structure. However, we cannot exclude the possibility that species 3 includes a $\mathrm{Cu}$ adatom (that is, $\mathrm{DAPyr}-\mathrm{Cu}$ complex); on-surface reactions can form metal-organic coordination complexes ${ }^{56}$ and AFM imaging has difficulty resolving the coordinated metal adatoms ${ }^{42,57}$.

Next, we discuss the skeletal rearrangement of DAPyr 4. It is noteworthy that the product yield of $\mathbf{5}$ is far larger than that of 6: 85 versus $2 \%$ at $T_{\text {anneal }}=245^{\circ} \mathrm{C}$ (see Fig. $3 \mathrm{~d}$ ). In other words, an azuleno moiety of $\mathrm{DAPh}$ on $\mathrm{Cu}(001)$ is converted predominantly into a fulvaleno moiety, whereas a conversion into a naphtho moiety rarely occurs. Such selective rearrangement of azulene derivatives will play an important role in the controllable creation and repair of Stone-Wales defects in graphene structures ${ }^{49,50}$. To our knowledge, in general, both azuleno and fulvaleno moieties are converted into a naphtho moiety by heat ${ }^{47-50,58}$ and no azulene-to-fulvalene rearrangement has been reported. This strongly indicates that the interaction with the substrate plays an important role in yielding the specific reaction product. Furthermore, previous experimental and theoretical studies have revealed that various reaction mechanisms coexist for the rearrangement of azulene to naphthalene in the gas phase $^{48-50}$. This suggests that multiple, coexisting reaction pathways are also conceivable for the reaction of 4 to $5 / 6$ on the surface. Based on the previous reports for the azulene-tonaphthalene rearrangement, we propose several possible reaction mechanisms for $\mathbf{4 \rightarrow 5 / 6}$ (see Supplementary Note 3 and Supplementary Figs 6 and 7).

Nonetheless, we can understand the mechanism of the selective reaction of $\mathbf{4} \rightarrow \mathbf{5}$ in a qualitative manner, by considering the correlation between the steric distortion and the reactivity of the molecules. In free space, as described above, the azuleno moieties of 4 are locally distorted by their mutual proximity (a molecular height of $2.2 \AA$; Fig. $4 \mathrm{~g}$ ), leading to a flattening energy of $171 \mathrm{meV}$ at a reaction temperature of $227^{\circ} \mathrm{C}$ (Table 1). A final product of the fulvaleno species 5 is planar even in free space (Fig. 4h). Therefore, the on-surface reaction of the distorted species 4 to yield the intrinsically planar product $\mathbf{5}$ proceeds exothermically to eliminate the strain instability in the molecule on the surface. The complete resolution of the geometric distortion may also be responsible for the fact that no reaction product with both azuleno moieties rearranged has been observed in our experiments. In contrast, the other product, that is, the naphtho species 6 , is still nonplanar (a molecular height of $1.0 \AA$ and a flattened energy of $110 \mathrm{meV}$; Fig. $4 \mathrm{i}$ ). The reaction of $\mathbf{4} \rightarrow \mathbf{6}$ is unfavourable because it retains the intramolecular strain, and 6 may be rearranged again into the totally planar product 5 .

Finally, we must also consider DAA 2, which cannot be rearranged. The flattening energy of DAA $2\left(104 \mathrm{meV}\right.$ at $227^{\circ} \mathrm{C}$; Table 1) is lower than that of DAPyr 4. Moreover, even though one of the azuleno moieties of DAA is rearranged into a fulvaleno moiety, this product should retain the intramolecular strain with a flattening energy is $86 \mathrm{meV}$ at $227^{\circ} \mathrm{C}$ (see Supplementary Note 2 and Supplementary Fig. 8). We should note that the order of the flattening energies may be much smaller than the reaction barrier for the skeletal rearrangement; indeed, the barrier for the azuleneto-naphthalene rearrangement in the gas phase is calculated to be above $1 \mathrm{eV}$ (refs 49,50). We assume that the strain energy of DAPyr 4 is enhanced due to interactions with the substrate such as charge transfer. To gain further insights into the reaction mechanism, theoretical calculations of the adsorption structures and reaction barriers would be helpful.

In summary, we have demonstrated on-surface skeletal rearrangement of an azulene-derivative $\mathrm{PAH}$ driven by the intramolecular structural strain. To derive a highly strained $\mathrm{PAH}$, we conducted a sequential procedure: organic synthesis of a $\mathrm{PAH}$ with two azuleno moieties in proximity to each other-that is, $\mathrm{DAPh} \mathbf{1 - a n d}$ thermal cyclodehydroganation of the molecule $\mathbf{1}$ adsorbed onto a $\mathrm{Cu}(001)$ surface. The product DAPyr $\mathbf{4}$ adopts a planar configuration on the surface, in spite of the fact that the azuleno moieties are sterically hindered by further mutual proximity. Therefore, the azuleno moieties flattened on the surface are highly strained. In order to eliminate the local strain instability, the molecule $\mathbf{4}$ is thermally induced to react predominantly into an intrinsically flat $\mathrm{PAH}$ with a fulvaleno moiety, which has never been yielded by conventional organic synthesis. The importance of the strain for the skeletal rearrangement is reinforced by the observation that a reference molecule-DAA 2-exhibits no thermal reactivity at the surface because it cannot eliminate the strain by any skeletal rearrangement. Such strain-induced reactions of organic molecules on surfaces would be a useful approach to synthesizing functional chemical products.

\section{Methods}

Experiments. The STM/AFM experiments were carried out in an ultrahighvacuum chamber (Omicron low-temperature STM/AFM system) at $4.8 \mathrm{~K}$. For frequency-modulation AFM, a tuning fork with an etched tungsten tip was used as a force sensor $^{59}$ (resonance frequency $f_{0}=21.4 \mathrm{kHz}$, spring constant $k_{0} \approx 1,800 \mathrm{~N} \mathrm{~m}^{-1}$, quality factor $Q \approx 2 \times 10^{4}$ ). For the AFM images, $\Delta f$ was measured in constant-height mode at a sample bias $V=0 \mathrm{~V}$ and an oscillation amplitude $A=2 \AA$, while the STM images were acquired in constant-current mode Single-crystalline $\mathrm{Cu}(001)$ was cleaned by repeated cycles of $\mathrm{Ar}^{+}$sputtering and annealing.

The methods of synthesis, purification and characterization for DAPh $\mathbf{1}$ and DAA 2 are described in Supplementary Methods and Supplementary Figs 9-16. These molecules were thermally sublimated from alumina crucibles at $\sim 130^{\circ} \mathrm{C}$ and deposited onto a clean surface at room temperature. The surface at $\sim 8 \mathrm{~K}$ was subsequently exposed to CO gas.

Calculations. The geometric optimizations of the molecules in free space were performed by Materials Studio $2016 \mathrm{DMol}^{3}$ B3LYP functional with a DNP basis set. The details of the calculations are described in Supplementary Methods.

Data availability. Supplementary crystallographic information files, which include structure factors, have been deposited with the Cambridge Crystallographic Data Centre (CCDC) as deposition numbers CCDC 1522349, 1; CCDC 1,522,350, 2. 
These data files can be obtained free of charge from http://www.ccdc.cam.ac.uk/ data_request/cif. The data that support the findings of this study are available from the corresponding author on reasonable request.

\section{References}

1. Wiberg, K. B. The concept of strain in organic chemistry. Angew. Chem. Int Ed. 25, 312-322 (1986).

2. Piotti, M. E. Ring opening metathesis polymerization. Curr. Opin. Solid State Mat. Sci. 4, 539-547 (1999).

3. Beyer, M. K. \& Clausen-Schaumann, H. Mechanochemistry: the mechanical activation of covalent bonds. Chem. Rev. 105, 2921-2948 (2005).

4. Ribas-Arino, J. \& Marx, D. Covalent mechanochemistry: theoretical concepts and computational tools with applications to molecular nanomechanics. Chem. Rev. 112, 5412-5487 (2012).

5. Wang, G. W. Mechanochemical organic synthesis. Chem. Soc. Rev. 42, 7668-7700 (2013)

6. Hickenboth, C. R. et al. Biasing reaction pathways with mechanical force. Nature 446, 423-427 (2007).

7. Alder, R. W. \& Harvey, J. N. Radical-promoted Stone-Wales rearrangements. J, Am. Chem. Soc. 126, 2490-2494 (2004).

8. Yakobson, B. I. Mechanical relaxation and 'intramolecular plasticity' in carbon nanotubes. Appl. Phys. Lett. 72, 918-920 (1998).

9. Yoon, M. et al. Zipper mechanism of nanotube fusion: theory and experiment. Phys. Rev. Lett. 92, 075504 (2004).

10. Carr, L. D. \& Lusk, M. T. Defect engineering: graphene gets designer defects. Nat. Nanotechnol. 5, 316-317 (2010).

11. Chuvilin, A., Kaiser, U., Bichoutskaia, E., Besley, N. A. \& Khlobystov, A. N. Direct transformation of graphene to fullerene. Nat. Chem. 2, 450-453 (2010).

12. Cataldo, F. The impact of a fullerene-like concept in carbon black science. Carbon 40, 157-162 (2002).

13. Scott, L. T. Thermal rearrangements of aromatic compounds. Acc. Chem. Res. 15, 52-58 (1982).

14. Narita, A., Wang, X.-Y., Feng, X. \& Mullen, K. New advances in nanographene chemistry. Chem. Soc. Rev. 44, 6616-6643 (2015).

15. Segawa, Y., Ito, H. \& Itami, K. Structurally uniform and atomically precise carbon nanostructures. Nat. Rev. Mater. 1, 15002 (2016).

16. Ciabini, L. et al. Triggering dynamics of the high-pressure benzene amorphization. Nat. Mater. 6, 39-43 (2007).

17. Fitzgibbons, T. C. et al. Benzene-derived carbon nanothreads. Nat. Mater. 14, 43-47 (2015).

18. Tsefrikas, V. M. \& Scott, L. T. Geodesic polyarenes by flash vacuum pyrolysis. Chem. Rev. 106, 4868-4884 (2006).

19. Jenkins, S. J. Aromatic adsorption on metals via first-principles density functional theory. Proc. R. Soc. London A 465, 2949-2976 (2009).

20. Maurer, R. J. et al. Adsorption structures and energetics of molecules on metal surfaces: Bridging experiment and theory. Prog. Surf. Sci. 91, 72-100 (2016).

21. Lafferentz, L. et al. Controlling on-surface polymerization by hierarchical and substrate-directed growth. Nat. Chem. 4, 215-220 (2012).

22. Shen, Q., Gao, H.-Y. \& Fuchs, H. Frontiers of on-surface synthesis: from principles to applications. Nano Today 13, 77-96 (2017).

23. Cai, J. et al. Atomically precise bottom-up fabrication of graphene nanoribbons. Nature 466, 470-473 (2010).

24. Björk, J., Stafström, S. \& Hanke, F. Zipping up: cooperativity drives the synthesis of graphene nanoribbons. J. Am. Chem. Soc. 133, 14884-14887 (2011).

25. Ruffieux, P. et al. On-surface synthesis of graphene nanoribbons with zigzag edge topology. Nature 531, 489-492 (2016).

26. Sakaguchi, H., Song, S., Kojima, T. \& Nakae, T. Homochiral polymerizationdriven selective growth of graphene nanoribbons. Nat. Chem. 9, 57-63 (2017).

27. Otero, G. et al. Fullerenes from aromatic precursors by surface-catalysed cyclodehydrogenation. Nature 454, 865-868 (2008).

28. Treier, M. et al. Surface-assisted cyclodehydrogenation provides a synthetic route towards easily processable and chemically tailored nanographenes. Nat. Chem. 3, 61-67 (2011)

29. Vasseur, G. et al. Quasi one-dimensional band dispersion and surface metallization in long-range ordered polymeric wires. Nat. Commun. 7, 10235 (2016).

30. Sanchez-Valencia, J. R. et al. Controlled synthesis of single-chirality carbon nanotubes. Nature 512, 61-64 (2014).

31. Stetsovych, O. et al. From helical to planar chirality by on-surface chemistry Nat. Chem. 9, 213-218 (2017).

32. Gross, L., Mohn, F., Moll, N., Liljeroth, P. \& Meyer, G. The chemical structure of a molecule resolved by atomic force microscopy. Science 325, 1110-1114 (2009).

33. Gross, L. Recent advances in submolecular resolution with scanning probe microscopy. Nat. Chem. 3, 273-278 (2011).

34. Jarvis, S. P. Resolving intra- and inter-molecular structure with non-contact atomic force microscopy. Int. J. Mol. Sci. 16, 19936-19959 (2015).

35. Pavliček, N. \& Gross, L. Generation, manipulation and characterization of molecules by atomic force microscopy. Nat. Rev. Chem. 1, 0005 (2017).
36. Shiotari, A. \& Sugimoto, Y. Ultrahigh-resolution imaging of water networks by atomic force microscopy. Nat. Commun. 8, 14313 (2017).

37. Pavliček, N. et al. Synthesis and characterization of triangulene. Nat. Nanotechnol. 12, 308-311 (2017).

38. Hapala, P. et al. Mechanism of high-resolution STM/AFM imaging with functionalized tips. Phys. Rev. B 90, 085421 (2014).

39. Hämäläinen, S. K. et al. Intermolecular contrast in atomic force microscopy images without intermolecular bonds. Phys. Rev. Lett. 113, 186102 (2014).

40. de Oteyza, D. G. et al. Direct imaging of covalent bond structure in singlemolecule chemical reactions. Science 340, 1434-1437 (2013).

41. Albrecht, F., Pavliček, N., Herranz-Lancho, C., Ruben, M. \& Repp, J. Characterization of a surface reaction by means of atomic force microscopy. J. Am. Chem. Soc. 137, 7424-7428 (2015).

42. Kocic, N. et al. Control of reactivity and regioselectivity for on-surface dehydrogenative aryl-aryl bond formation. J. Am. Chem. Soc. 138, 5585-5593 (2016).

43. Krüger, J. et al. Tetracene formation by on-surface reduction. ACS Nano 10, 4538-4542 (2016).

44. Riss, A. et al. Imaging single-molecule reaction intermediates stabilized by surface dissipation and entropy. Nat. Chem. 8, 678-683 (2016).

45. He, Y. et al. Fusing tetrapyrroles to graphene edges by surface-assisted covalent coupling. Nat. Chem. 9, 33-38 (2017).

46. Kawai, S. et al. Thermal control of sequential on-surface transformation of a hydrocarbon molecule on a copper surface. Nat. Commun. 7, 12711 (2016).

47. Gordon, M. The azulenes. Chem. Rev. 50, 127-200 (1952).

48. Scott, L. T. \& Kirms, M. A. Azulene thermal rearrangements. Carbon-13 labeling studies of automerization and isomerization to naphthalene. J. Am. Chem. Soc. 103, 5875-5879 (1981).

49. Alder, R. W., East, S. P., Harvey, J. N. \& Oakley, M. T. The azulene-tonaphthalene rearrangement revisited: A DFT study of intramolecular and radical-promoted mechanisms. J. Am. Chem. Soc. 125, 5375-5387 (2003).

50. Stirling, A., Iannuzzi, M., Laio, A. \& Parrinello, M. Azulene-to-naphthalene rearrangement: the car-parrinello metadynamics method explores various reaction mechanisms. ChemPhysChem 5, 1558-1568 (2004).

51. Dahlgren, D. \& Hemminger, J. C. Chemisorption and thermal chemistry of azulene and naphthalene adsorbed on Pt(111). Surf. Sci. 114, 459-470 (1982).

52. Hallmark, V., Chiang, S., Meinhardt, K.-P. \& Hafner, K. Observation and calculation of internal structure in scanning tunneling microscopy images of related molecules. Phys. Rev. Lett. 70, 3740 (1993).

53. Boneschanscher, M. P., Hämäläinen, S. K., Liljeroth, P. \& Swart, I. Sample corrugation affects the apparent bond lengths in atomic force microscopy. ACS Nano 8, 3006-3014 (2014).

54. Iwata, K. et al. Chemical structure imaging of a single molecule by atomic force microscopy at room temperature. Nat. Commun. 6, 7766 (2015).

55. Majzik, Z. et al. Synthesis of a naphthodiazaborinine and its verification by planarization with atomic force microscopy. ACS Nano 10, 5340-5345 (2016)

56. Stepanow, S., Lin, N. \& Barth, J. V. Modular assembly of low-dimensional coordination architectures on metal surfaces. J. Phys.: Condens. Matter 20, 184002 (2008).

57. Kawai, S. et al. Organometallic bonding in an Ullmann-type on-surface chemical reaction studied by high-resolution atomic force microscopy. Small 12, 5303-5311 (2016).

58. Irace, E. E., Brayfindley, E., Vinnacombe, G. A., Castro, C. \& Karney, W. L. Stone-Wales rearrangements in hydrocarbons: from planar to bowl-shaped substrates. J. Org. Chem. 80, 11718-11725 (2015).

59. Giessibl, F. J. High-speed force sensor for force microscopy and profilometry utilizing a quartz tuning fork. Appl. Phys. Lett. 73, 3956-3958 (1998).

\section{Acknowledgements}

This work was supported by JSPS KAKENHI Grant Numbers JP16H00959, JP25110003, JP16H00967, JP15H00995, JP15H06127, JP16K17893, JP16K13680, JP16H00933, JP15H03566 and JP14J05691; 'Zero-Emission Energy Research' of the International Energy Agency, Kyoto University. Y.S. acknowledges the support of the Iketani Science and Technology Foundation, the Noguchi Institute, the Japan Association for Chemica Innovation and the Murata Science Foundation. Super Computer System, Institute for Chemical Research, Kyoto University was used for the calculations. A.S., K.I. and Y.S. thank T. Osaka for technical assistance. T.N., S.M., T.O. and H.U. thank Y. Shinagawa and T. Kikuchi for technical assistance.

\section{Author contributions}

A.S. and T.N. contributed equally to this work. Y.S. and H.U. designed the experiments A.S. and K.I. performed the STM and AFM measurements. T.N., S.M. and T.O. synthesized DAPh and DAA molecules. T.N., S.M. and H.U. performed X-ray analysis. T.N. conducted the theoretical calculations. Y.S., H.S. and H.U. directed the project. A.S. analysed the data and wrote the main text. A.S. and T.N. wrote the Supplementary Information. All authors discussed the results and commented on the manuscript. 


\section{Additional information}

Supplementary Information accompanies this paper at http://www.nature.com/ naturecommunications

Competing interests: The authors declare no competing financial interests.

Reprints and permission information is available online at http://npg.nature.com/ reprintsandpermissions/

How to cite this article: Shiotari, A. et al. Strain-induced skeletal rearrangement of a polycyclic aromatic hydrocarbon on a copper surface. Nat. Commun. 8, 16089 doi: 10.1038/ncomms16089 (2017).

Publisher's note: Springer Nature remains neutral with regard to jurisdictional claims in published maps and institutional affiliations. cc (i) Open Access This article is licensed under a Creative Commons Attribution 4.0 International License, which permits use, sharing, adaptation, distribution and reproduction in any medium or format, as long as you give appropriate credit to the original author(s) and the source, provide a link to the Creative Commons license, and indicate if changes were made. The images or other third party material in this article are included in the article's Creative Commons license, unless indicated otherwise in a credit line to the material. If material is not included in the article's Creative Commons license and your intended use is not permitted by statutory regulation or exceeds the permitted use, you will need to obtain permission directly from the copyright holder. To view a copy of this license, visit http://creativecommons.org/ licenses/by/4.0/

(C) The Author(s) 2017 